\title{
LRRC36 is differentially expressed in non-small cell lung cancer and associates with
}

patient survival.

Shahan Mamoor, MS ${ }^{1}$

1shahanmamoor@gmail.com

East Islip, NY USA

Non-small cell lung cancer (NSCLC) is the leading cause of cancer death in the United States ${ }^{1}$. We mined published microarray data $2,3,4$ to identify differentially expressed genes in NSCLC.

We found that the gene encoding LRRC36 was among those whose expression was most different in human NSCLC tumors as compared to the lung. LRRC36 expression levels were significantly decreased in NSCLC tumors as compared to the lung, and lower expression of LRRC36 in patient tumors was significantly associated with worse overall survival. LRRC36 may be important for initiation or progression of non-small cell lung cancer in humans.

Keywords: LRRC36, NSCLC, non-small cell lung cancer, systems biology of NSCLC, targeted therapeutics in NSCLC.

1 OF 14 
In 2016, lung cancer resulted in the death of 158,000 Americans; $81 \%$ of all patients diagnosed with lung cancer will expire within 5 years 5 . Non-small cell lung cancer (NSCLC) is the most common type of lung cancer, diagnosed in $84 \%$ of patients with lung cancer, and $76 \%$ of all patients with NSCLC will expire within 5 years 5 . The rational development of targeted therapeutics to treat patients with NSCLC can be supported by an enhanced understanding of fundamental transcriptional features of NSCLC tumors. To discover genes associated with NSCLC tumors in an unbiased fashion and at the systems-level, we mined independently published microarray data $2,3,4$ to compare global gene expression profiles of NSCLC tumors to that of the normal lung. We found recurrent and significant differential expression of LRRC36 in adenocarcinoma tumors from patients with NSCLC, suggesting LRRC36 may be important for NSCLC tumor initiation or progression.

\section{Methods}

We utilized microarray datasets, GSE43458², GSE335324² and GSE747064 for this differential gene expression analysis of NSCLC tumors in conjunction with GEO2R. GSE43458 was generated using Affymetrix Human Gene 1.0 ST Array technology; for this analysis, we used with $n=30$ control lung tissue and $n=80$ NSCLC tumors, and the analysis was performed using platform GPL6244. GSE33532 was generated using Affymetrix Human Genome U133 Plus 2.0 Array technology; for this analysis, we used $n=20$ control lung tissue and $n=10$ NSCLC tumors, and the analysis was performed using platform GPL570. GSE74706 was generated using Agilent-026652 Whole Human Genome Microarray 4x44K v2 technology; for this 
analysis, we used $n=18$ control lung tissue and $n=10$ NSCLC tumors, and the analysis was performed using platform GPL13497. All tumors utilized for differential gene expression analysis here were of the adenocarcinoma type.

The Benjamini and Hochberg method of $p$-value adjustment was used for ranking of differential expression but raw $p$-values were used to assess statistical significance of global differential expression. Log-transformation of data was auto-detected, and the NCBI generated category of platform annotation was used. A statistical test was performed to evaluate whether LRRC36 expression was significantly between normal lung tissue and NSCLC tumors using a two-tailed, unpaired t-test with Welch's correction. We used PRISM for all statistical analyses of differential gene expression in NSCLC tumors (Version 8.4.0)(455). For KaplanMeier survival analysis, we used the Kaplan-Meier plotter online tool ${ }^{5}$ for correlation of LRRC36 mRNA expression levels with overall survival in $n=1925$ non-small cell lung cancer patients.

\section{Results}

We harnessed the power of multiple, independently published microarray datasets $2,3,4$ to discover in an unbiased fashion and at the transcriptome-level the most striking gene expression features of NSCLC tumors.

LRRC36 is differentially expressed in non-small cell lung cancers.

We found significant differential expression of LRRC36 in NSCLC tumors when compared to the lung2 (Table 1 ). When sorting each of the transcripts measured by microarray 
based on significance of difference in LRRC36 expression between NSCLC tumors and the normal lung, in this dataset, LRRC36 ranked 248 out of 25906 total transcripts, equating to 99.0\% differential expression (Table 1). Differential expression of LRRC36 in NSCLC tumors was statistically significant (Table $1 ; p=6.29 \mathrm{E}-20$ ).

We queried a second microarray dataset ${ }^{3}$ to determine if we could validate differential expression of LRRC36 in non-small cell lung cancers (Table 2). We found significant differential expression of LRRC36 in NSCLC tumors of the adenocarcinoma type when compared to the normal lung (Table 2). When sorting each of the transcripts measured based on significance of difference in expression of LRRC36 between NSCLC tumors and the normal lung, LRRC36 ranked 45 of 33252 total transcripts (Table 2), equating to $99.9 \%$ differential expression. Differential expression of LRRC36 in NSCLC tumors was statistically significant (Table 2; $p=2.68 \mathrm{E}-16$ ).

Analysis of a third microarray dataset ${ }^{4}$ again revealed significant differential expression of LRRC36 in NSCLC tumors of the adenocarcinoma type. When sorting each of the transcripts measured based on significance of difference in expression of LRRC36 between NSCLC tumors and the normal lung, LRRC36 ranked 793 out of 34183 total transcripts, equating to $97.7 \%$ differential expression (Table 3). Differential expression of LRRC36 in NSCLC tumors was statistically significant (Table $3 ; p=1.29 \mathrm{E}-08)$.

LRRC36 is expressed at significantly lower levels in NSCLC tumors as compared to the lung. We obtained exact mRNA levels for LRRC36 from NSCLC tumors and from the lung to 
directly compare LRRC36 expression between tumor and control lung tissue and assess for statistical significance. LRRC36 was expressed at significantly lower levels in NSCLC tumors as compared to the normal lung in each dataset queried, respectively (Figure 1: $p<0.0001$, Figure 2: $p<0.0001$, and Figure 3: $p<0.0001$ ). We calculated a mean fold change of $0.8024 \pm 0.0874$ and $0.6090 \pm 0.0253$ in LRRC36 expression when comparing NSCLC tumors to the lung (Table 1 and Table 2, respectively).

LRRC36 expression in NSCLC tumors correlates with overall survival.

We performed Kaplan-Meier survival analysis using LRRC36 mRNA expression in NSCLC tumors coupled with paired overall survival data from each patient, in 1925 NSCLC patients in total, to determine whether LRRC36 tumor expression was correlated with survival outcomes in NSCLC. We found that patients whose tumors expressed lower levels of LRRC36 possessed significantly shorter overall survival than patients with high tumor expression of LRRC36 (Figure 4). Median overall survival (OS) of patients in the low expression cohort was 57.33 months, while median OS in patients in the high LRRC36 expression cohort was 105.4 months (Table 4); this difference in median OS based on LRRC36 tumor expression in NSCLC was statistically significant (Figure 4; logrank $p$-value: 1.6e-9: hazard ratio: 0.61 (0.52 - 0.72); false discovery rate $=0.01$ ).

Thus, blind comparative transcriptome analysis of non-small cell lung cancers revealed differential expression of transcripts encoded by the LRRC36 gene as among the most significant transcriptional features of NSCLC tumors, and LRRC36 expression was significantly correlated 
with patient outcomes, as patients with lower tumor expression of LRRC36 possessed significantly worse overall survival.

\section{Discussion}

We could find little information describing functions for LRRC36, in normal biology or in cancer. LRRC36 is located at $16 \mathrm{q} 22.1$ and has been described as uncharacterized, but is phylogenetically most related to the centriolar satellite protein Cep727. LRRC36 contains a leucine-rich repeat (LRR) at the amino terminus and a coiled-coil domain at the carboxyterminus7. In mammalian cells in vitro, an LRRC36-green fluorescent protein (GFP) fusion localizes to the centrosome. In a study of 186 primary tumors of the colon and 54 liver metastases, LRRC36 was found to be significantly up-regulated in both primary tumors and liver metastases $^{8}$.

We found, by mining multiple independently published microarray datasets, that a relatively uncharacterized molecule, LRRC36, was among the genes most differentially expressed in the primary tumors of patients with non-small cell lung cancer; LRRC36 was expressed at significantly lower levels in NSCLC tumors as compared to the lung, and decreased expression of LRRC36 was significantly associated with worse overall survival in NSCLC patients. LRRC36 may be of relevance to the biology of tumor initiation or progression in patients with NSCLC of the adenocarcinoma type, the most common type of the leading cause of cancer death in the United States and worldwide.

6 OF 14 


\section{References}

1. Siegel, R.L., Miller, K.D. and Jemal, A., 2019. Cancer statistics, 2019. CA: a cancer journal for clinicians, 69(1), pp.7-34.

2. Meister, M., Belousov, A., Xu, E.C., Schnabel, P., Warth, A. and Hoofmann, H., 2014. Intratumor heterogeneity of gene expression profiles in early stage non-small cell lung cancer. $\mathrm{J}$ Bioinf Res Stud, 1, p.1.

3. Marwitz, S., Depner, S., Dvornikov, D., Merkle, R., Szczygieł, M., Müller-Decker, K., Lucarelli, P., Wäsch, M., Mairbäurl, H., Rabe, K.F. and Kugler, C., 2016. Downregulation of the TGF $\beta$ pseudoreceptor BAMBI in non-small cell lung cancer enhances TGF $\beta$ signaling and invasion. Cancer research, 76(13), pp.3785-3801.

4. Kabbout, M., Garcia, M.M., Fujimoto, J., Liu, D.D., Woods, D., Chow, C.W., Mendoza, G., Momin, A.A., James, B.P., Solis, L. and Behrens, C., 2013. Ets2 mediated tumor suppressive function and met oncogene inhibition in human non-small cell lung cancer. Clinical cancer research, 19(13), pp.3383-3395.

5. Lung Cancer - Non-Small Cell: Statistics. https:/www.cancer.net/cancer-types/lung-cancernon-small-cell/statistics.

6. Gyorffy, B., Surowiak, P., Budczies, J. and Lanczky, A., 2013. Online survival analysis software to assess the prognostic value of biomarkers using transcriptomic data in non-smallcell lung cancer. PloS one, 8(12), pp.e82241-e82241.

7. Stowe, T.R., Wilkinson, C.J., Iqbal, A. and Stearns, T., 2012. The centriolar satellite proteins Cep72 and Cep290 interact and are required for recruitment of BBS proteins to the cilium. Molecular biology of the cell, 23(17), pp.3322-3335.

8. Qian, Z., Zhang, G., Song, G., Shi, J., Gong, L., Mou, Y. and Han, Y., 2017. Integrated analysis of genes associated with poor prognosis of patients with colorectal cancer liver metastasis. Oncotarget, 8(15), p.25500.

7 OF 14 


\begin{tabular}{|c|c|c|c|c|c|c|c|c|}
\hline Rank & ID & p-value & $\mathbf{t}$ & B & FC & Gene & Gene name & $\%$ DE \\
\hline 248 & 7996546 & 6.29E-20 & -11.189446 & 34.79623 & $\begin{array}{l}0.8024 \pm \\
0.0874\end{array}$ & LRRC36 & $\begin{array}{l}\text { leucine rich repeat } \\
\text { containing } 36\end{array}$ & 99.0 \\
\hline
\end{tabular}

Table 1: LRRC36 is differentially expressed in NSCLC tumors.

The rank of differential expression relative all transcripts measured, probe ID, $p$-value of global differential expression, $t$, a moderated t-statistic, $\mathrm{B}$, the log-odds of differential expression between the groups compared, fold change of LRRC36 expression in NSCLC tumors as compared to the lung, gene, gene name and percent differential expression (\% DE) are listed in this chart. 
9 OF 14 
10 OF 14 


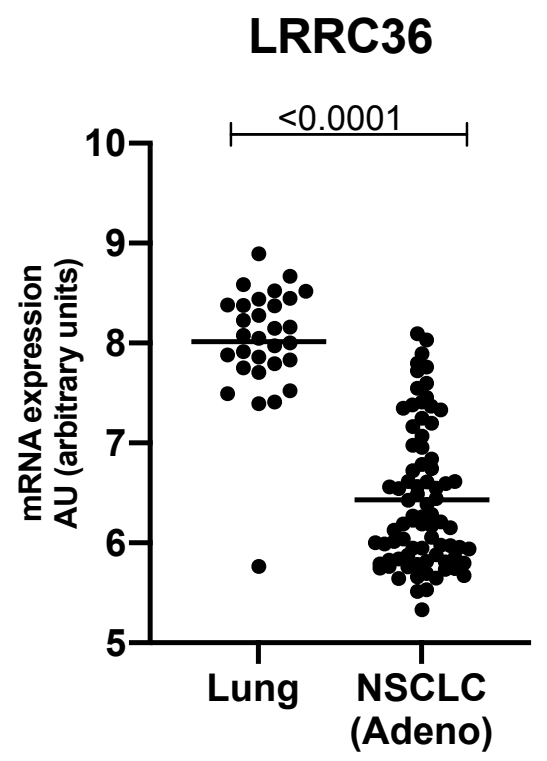

Figure 1: LRRC36 is expressed at significantly lower levels in NSCLC tumors when compared to the lung.

The mRNA expression level of LRRC36 is graphically represented in the lung (left) and in NSCLC tumors of the adenocarcinoma type (right) with mean mRNA expression values marked and the result of a statistical test evaluating significance of difference in LRRC36 expression between NSCLC tumors and the lung, a $p$-value, listed above.

11 OF 14 


\section{LRRC36}

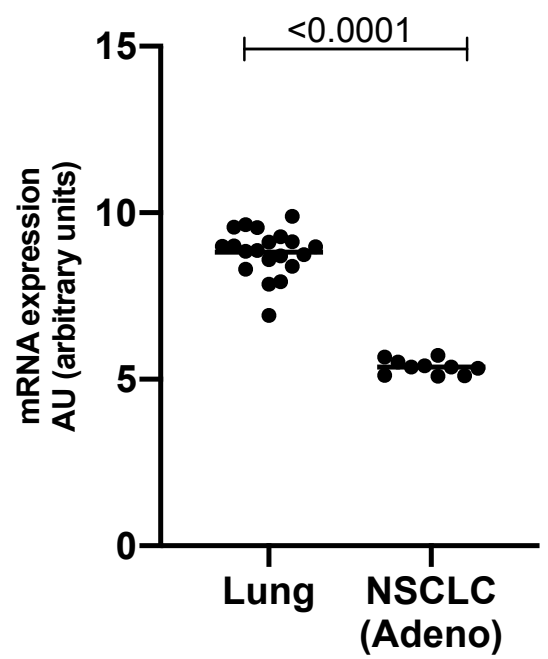

Figure 2: LRRC36 is expressed at significantly lower levels in NSCLC tumors when compared to the lung.

The mRNA expression level of LRRC36 is graphically represented in the lung (left) and in NSCLC tumors of the adenocarcinoma type (right) with mean mRNA expression values marked and the result of a statistical test evaluating significance of difference in LRRC36 expression between NSCLC tumors and the lung, a $p$-value, listed above. 


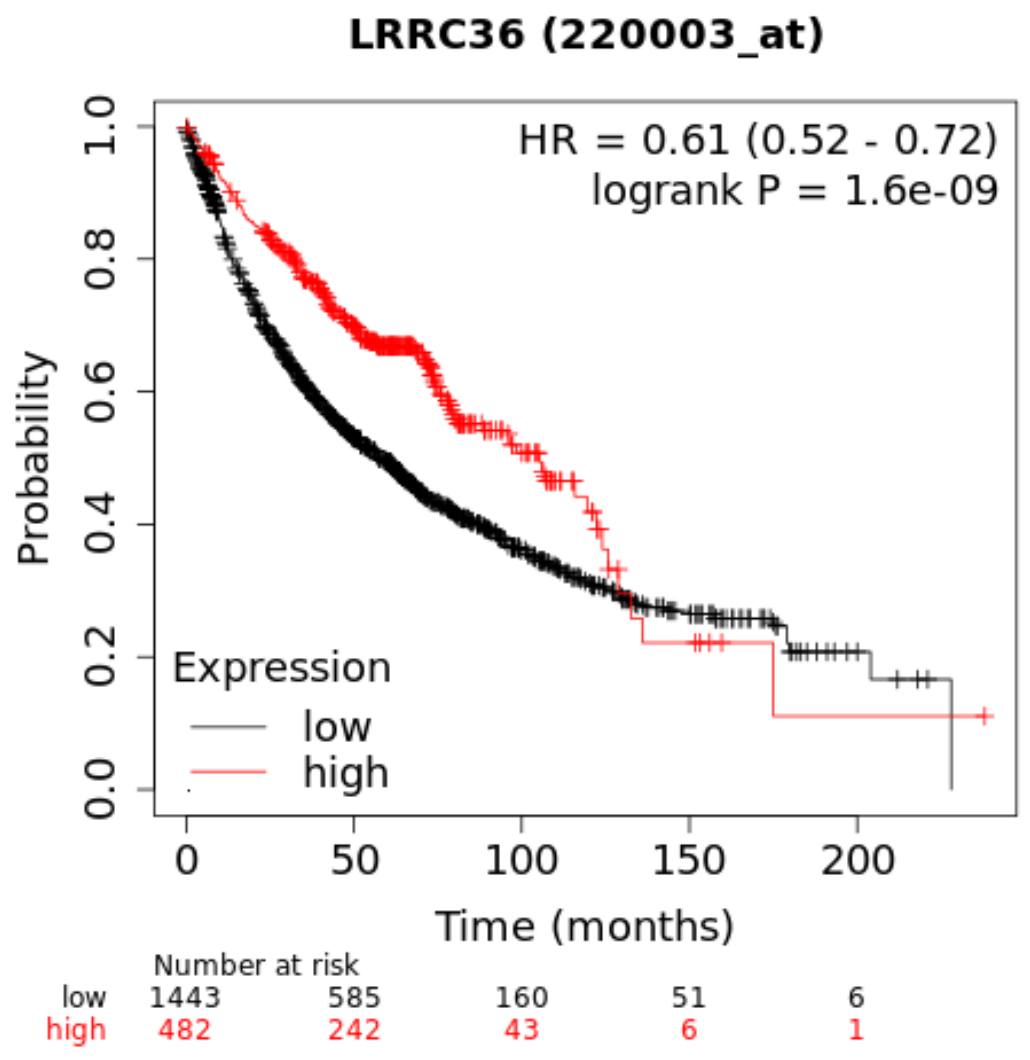

Figure 3: LRRC36 expression in NSCLC tumors significantly correlates with overall survival.

Depicted in this Kaplan-Meier plot is the probability of overall survival for $n=1925$ total patients stratified into two groups, based on low or high expression of LRRC36 in patient tumors. The $\log$ rank $p$-value denoting statistical significance of difference in overall survival when comparing the two groups, as well as hazard ratio for this comparison is listed above. Listed below is the number of patients at risk (number of patients alive) per interval, after stratification based on LRRC36 expression; in the first interval, number at risk is number of patients alive; in each subsequent interval, number at risk is the number at risk less those who have expired or are censored. 
14 OF 14 